\title{
Investigation of PACAP Fragments and Related Peptides in Chronic Retinal Hypoperfusion
}

\author{
Dora Werling, ${ }^{1}$ Dora Reglodi, ${ }^{1}$ Peter Kiss, ${ }^{1}$ Gabor Toth, ${ }^{2}$ Krisztina Szabadfi, ${ }^{3}$ \\ Andrea Tamas, ${ }^{1}$ Zsolt Biro, ${ }^{4}$ and Tamas Atlasz ${ }^{1,5,6}$ \\ ${ }^{1}$ Department of Anatomy, PTE-MTA “Lendulet” PACAP Research Team, University of Pecs, Szigeti ut 12, Pecs 7624, Hungary \\ ${ }^{2}$ Department of Medical Chemistry, University of Szeged, Dom Ter 8, Szeged 6720, Hungary \\ ${ }^{3}$ Department of Experimental Zoology and Neurobiology, University of Pecs, Ifjusag Utja 6, Pecs 7624, Hungary \\ ${ }^{4}$ Department of Ophthalmology, University of Pecs, Nyar Utca 8, Pecs 7624, Hungary \\ ${ }^{5}$ Department of Sportbiology, University of Pecs, Ifjusag Utja 6, Pecs 7624, Hungary \\ ${ }^{6}$ Janos Szentagothai Research Center, University of Pecs, Ifjusag Utja 20, Pecs 7624, Hungary
}

Correspondence should be addressed to Tamas Atlasz; attam@gamma.ttk.pte.hu

Received 20 January 2014; Accepted 24 March 2014; Published 12 May 2014

Academic Editor: Robert Gabriel

Copyright (C) 2014 Dora Werling et al. This is an open access article distributed under the Creative Commons Attribution License, which permits unrestricted use, distribution, and reproduction in any medium, provided the original work is properly cited.

Pituitary adenylate cyclase activating polypeptide (PACAP) has neuroprotective effects in different neuronal and retinal injuries. Retinal ischemia can be effectively modelled by permanent bilateral common carotid artery occlusion (BCCAO), which causes chronic hypoperfusion-induced degeneration in the entire rat retina. The retinoprotective effect of PACAP 1-38 and VIP is wellestablished in ischemic retinopathy. However, little is known about the effects of related peptides and PACAP fragments in ischemic retinopathy. The aim of the present study was to investigate the potential retinoprotective effects of different PACAP fragments (PACAP 4-13, 4-22, 6-10, 6-15, 11-15, and 20-31) and related peptides (secretin, glucagon) in BCCAO-induced ischemic retinopathy. Wistar rats (3-4 months old) were used in the experiment. After performing BCCAO, the right eyes of the animals were treated with PACAP fragments or related peptides intravitreal $(100 \mathrm{pM})$, while the left eyes were injected with saline serving as control eyes. Sham-operated (without BCCAO) rats received the same treatment. Routine histology was performed 2 weeks after the surgery; cells were counted and the thickness of retinal layers was compared. Our results revealed significant neuroprotection by PACAP 1-38 but did not reveal retinoprotective effect of the PACAP fragments or related peptides. These results suggest that PACAP 1-38 has the greatest efficacy in ischemic retinopathy.

\section{Introduction}

Pituitary adenylate cyclase activating polypeptide (PACAP) is a neuropeptide with widespread occurrence in various organs and diverse effects both in the nervous system and in the periphery $[1,2]$. PACAP is strongly expressed in the central nervous system, where it exerts several effects such that it is a central regulator of circadian rhythmic activities [3], plays a role in memory formation [4] and psychiatric processes [5], and is involved in central feeding control [6].

PACAP is also known to be expressed in the retina, along with its receptors (PAC1, VPAC1, and VPAC2 receptors).
Numerous studies have provided evidence that the neuroprotective effects are mainly mediated by the $\mathrm{PACl}$ receptor and diverging downstream pathways upon its activation [79]. The PACl receptor has several splice variants, which can mediate not only different but also opposing effects [10]. Several research groups have proven that PACAP has strong protective effects against various retinal injuries. In vitro, it protects retinal explants against excitotoxic injury [11] and retinal pigment epithelial cells against oxidative stress [12]. In vivo retina studies show that PACAP protects against NMDAand MSG-induced excitotoxic damage [13, 14], UV lightinduced lesion [15], and optic nerve lesion [16]. Recent studies 
show that PACAP is also protective in diabetic retinopathy [17]. Retinal ischemia can be induced by several methods, mimicking pathological features seen in human glaucomarelated retinal lesions, in chronic retinal hypoperfusion, and in other types of retinal lesions accompanied by ischemia. The protective role of PACAP has also been proven in models of retinal ischemia $[18,19]$. Based on these studies, evidence for the protective effects of PACAP in the retina is now well established [20].

The bioavailability and fast degradation of PACAP limit its therapeutic use and attention has been drawn to the application of shorter fragments and/or analogs. N-terminally shorter fragments of PACAP usually have antagonistic effects, but some reports have documented agonistic behavior, depending on the cell/tissue type $[7-9,21,22]$. In contrast, C-terminally shorter fragments usually differ in the strength of receptorial binding [7-9]. Therefore, it is necessary to test whether shorter PACAP fragments have any effect, ameliorating or damaging, on retinal lesions. We have previously shown that PACAP 6-38, the most widely used antagonist of PACAP, has an aggravating effect on retinal excitotoxic lesion [23]. However, it is not known, whether the shorter fragments of PACAP have any effect on the retina. Therefore, the first aim of our present study was to examine the effects of PACAP fragments 4-13, 4-22, 6-10, 6-15, 11-15, and 20-31 on chronic retinal hypoperfusion induced by bilateral carotid artery occlusion. Possible fragments to be tested were selected in order to cover a wide range of the molecule, from the $\mathrm{N}$ terminal to the C-terminal and middle region of the peptide. In addition, the $\mathrm{N}$-terminal fragments show a high similarity with the structure of VIP. Furthermore, the 4-13 domain, for example, shows high selectivity to the PAC1 receptor, which is mainly responsible for the neuroprotective effects of PACAP [7-9]. In a recent study we have shown that the peptide most closely related to PACAP, namely, vasoactive intestinal peptide (VIP), is also protective in retinal ischemia. However, to achieve a degree of neuroprotection similar to PACAP, higher doses are required [24]. Other members of the peptide family have not been tested in retinal ischemia so far. Therefore, the second aim of the present study was to investigate whether secretin and glucagon have any effect on a rat model of retinal ischemia.

\section{Materials and Methods}

Experimental animals were derived from a local colony of Wistar rats. Animals were housed in individual cages, fed, and watered ad libitum, under light/dark cycles of 12/12 h. All animal procedures complied with the University of Pecs (number BA02/2000-15024/2011) for the ethical use of animals. Adult male rats $(n=32)$ weighing $250-300 \mathrm{~g}$ were exposed to permanent bilateral common carotid artery occlusion (BCCAO) under isoflurane anesthesia and both common carotid arteries were ligated with a 3-0 filament through a midline incision [19]. A group of animals underwent anesthesia and all steps of the surgical procedure, except ligation of the carotid arteries. These animals served as sham-operated animals $(n=7)$. Immediately following the operation, PACAP 1-38 or its fragments (PACAP 4-13, $4-22,6-10,6-15,11-15$, and 20-31) or other members of the peptide family such as secretin and glucagon $(100 \mathrm{pmol} / 5 \mu \mathrm{L})$ were injected intravitreally using 30 -gauge Hamilton syringe into the right vitreous body of animals. The left eyes received the same volumes of vehicle treatment (physiological saline) and served as ischemic eyes. Rats were sacrificed with an overdose of anesthetic after 2 weeks of BCCAO and the eyes were processed for histological analysis. Briefly, the eyes were dissected in PBS and fixed in 4\% paraformaldehyde dissolved in $0.1 \mathrm{M}$ PB. Following fixation, tissues were embedded in Durcupan ACM resin, cut at $2 \mu \mathrm{m}$, and stained with toluidine blue. Four tissue blocks from at least four animals were prepared and central retinal areas within $1 \mathrm{~mm}$ from the optic nerve were used for measurements $(n=5$ measurements from one tissue block). Photographs were taken with a digital CCD camera using the Spot program. Sections where the GCL appeared thicker than a single cell row were omitted from evaluation. The following parameters were measured: (i) the width of the outer and inner nuclear and plexiform layers (ONL, OPL, INL, and IPL, resp.); (ii) the number of cells $/ 100 \mu \mathrm{m}$ section length in the ganglion cell layer (GCL); (iii) the number of cells $/ 500 \mu \mathrm{m}^{2}$ area in the INL. Results are presented as mean \pm SEM. Statistical comparisons were made using the ANOVA test followed by Tukey-B's post hoc analysis.

\section{Results and Discussion}

BCCAO resulted in severely reduced thickness of retinal layers as observed two weeks after ligation compared to sham-operated controls (Figure 1(a)). Marks of degeneration with individual variations are visible in all retinal layers (Figure 1(b)). Morphometric analysis revealed that the most pronounced reduction in thickness in retinas with BCCAO was found in the OPL (Figure 2). The photoreceptor layer was also reduced in thickness (Figure 1(b)). This layer was significantly thinner than that of the control specimens (Figure 2). Many cells in the ganglion cell layer (GCL) also suffered degeneration, shown by necrotic cells in this layer (Figures 1 and 2). This fact is well reflected in the reduced number of cells in the GCL (Figure 2). Intraocular PACAP 1-38 treatment following BCCAO led to nearly healthy appearance of the retinal layers (Figure 1(c)). This is also well supported by the morphometric measurements. The thickness of the major retinal layers was almost identical with that of the sham-operated animals and was significantly larger than that of control ischemic ones (Figure 2). This was especially conspicuous in the OPL, which disappeared, and the ONL and INL layers were fused in several control animals and were preserved in all PACAP 1-38 injected animals. However, the number of cells in the GCL seemed to be lower than in the sham-operated animals (Figure 2). Indeed, some degenerating cells could be discerned in the GCL in these preparations. This may have led to a decrease in cell numbers.

Intravitreal injection of different PACAP fragments (PACAP 4-22, 6-15, 11-15, 20-31, 6-10, and 4-13) did not 


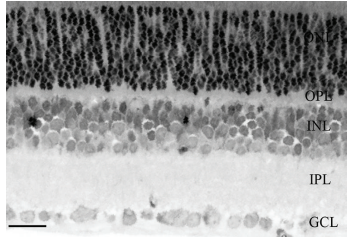

(a)

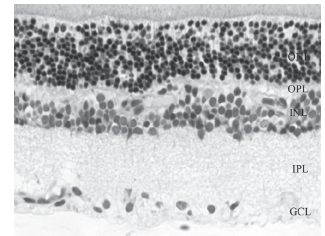

(d)

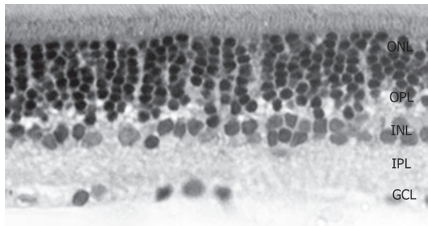

(g)

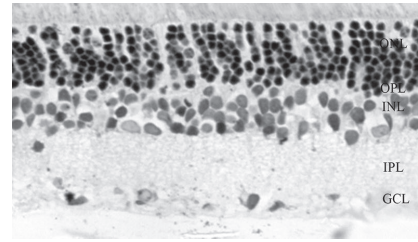

(b)

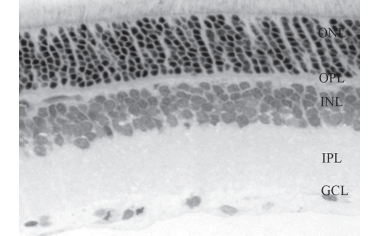

(c)

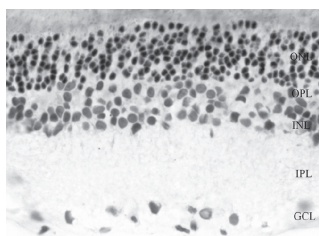

(e)

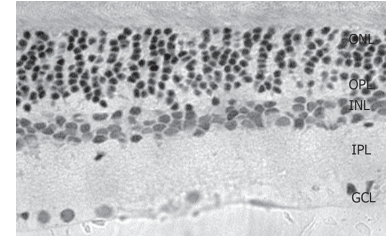

(h)

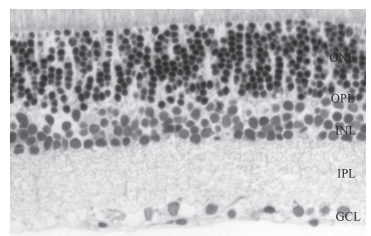

(f)

FIGURE 1: Representative light microphotographs of retinal sections. Retinal tissue from SHAM-operated animal (a) compared to BCCAO retinas (b). Representative sections from retinas treated with PACAP 1-38 (c) and PACAP fragments: PACAP 4-22 (d), 6-15 (e), 11-15 (f), 20-31 (g), 6-10 (h), and 4-13 (i). In contrast to PACAP 1-38 (c), the PACAP fragments (d-f) did not ameliorate the BCCAO-induced retinal degeneration. On the contrary, treatment with some fragments led to a more pronounced degeneration, which was shown by the width of the retinal layers and the neural profiles in the ONL and INL. ONL: outer nuclear layer; OPL: outer plexiform layer; INL: inner nuclear layer; IPL: inner plexiform layer; GCL: ganglion cell layer (scale bar: $20 \mu \mathrm{m}$ ).

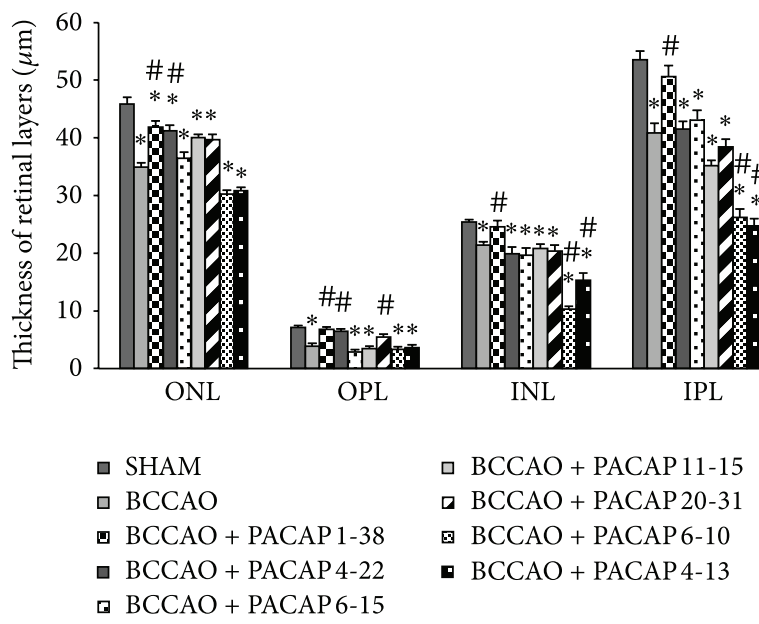

(a)

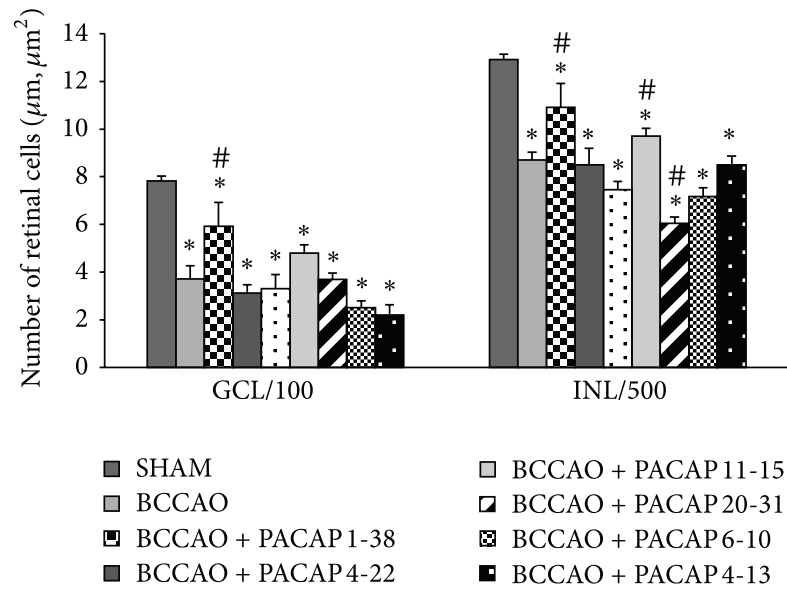

(b)

FIGURE 2: Morphometric analysis of retinal layers (a) and number of cells/100 $\mu \mathrm{m}$ GCL length and number of cells $/ 500 \mu \mathrm{m}^{2}$ INL (b) in SHAM operated animals, with BCCAO and with BCCAO + different PACAP fragments. We did not detect retinoprotective effects after the injection of the PACAP fragments (PACAP 4-22, 6-15, 11-15, 20-31, 6-10, and 4-13). ONL: outer nuclear layer; OPL: outer plexiform layer; INL: inner nuclear layer; IPL: inner plexiform layer; GCL: ganglion cell layer. ${ }^{*} P<0.05$ compared to SHAM retinas; ${ }^{\#} P<0.05$ compared to BCCAO retinas. 


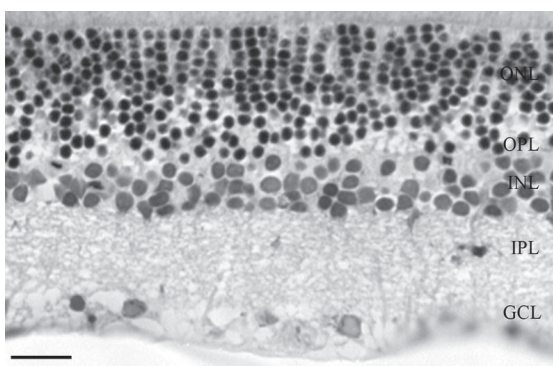

(a)

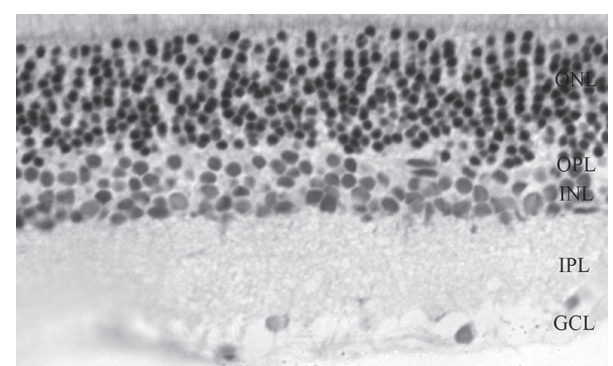

(b)

FIGURE 3: Light microphotographs of retinal sections (toluidine blue). Representative retinas from $\mathrm{BCCAO}+$ secretin (a) and $\mathrm{BCCAO}+$ glucagon (b). The whole retina structure (especially ONL and INL) was damaged by BCCAO compared to SHAM retinas. The PACAP related peptide secretin (a) and glucagon (b) did not ameliorate the ischemic degeneration (scale bar: $20 \mu \mathrm{m}$ ).

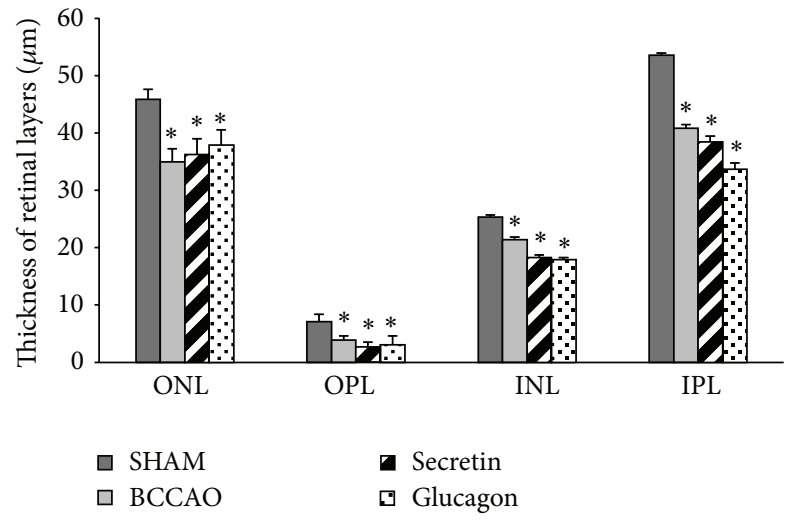

(a)

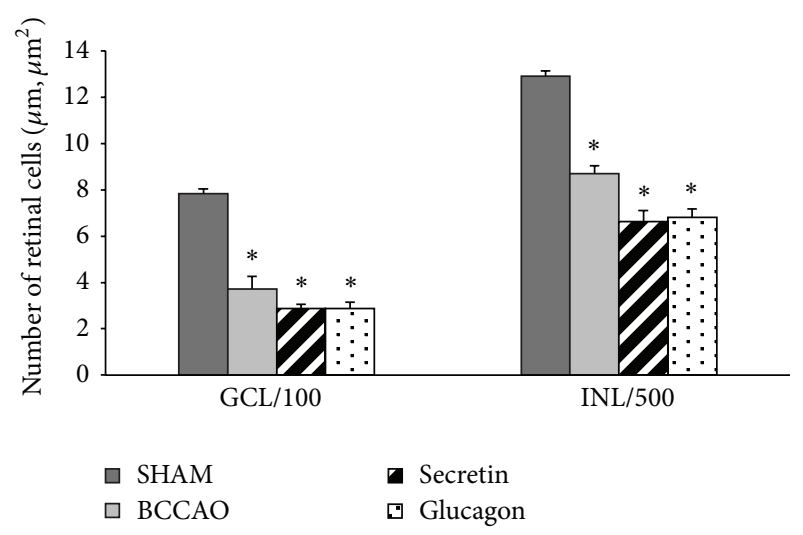

(b)

FIGURE 4: Morphometric analysis of retinal layers (a) and number of cells $/ 100 \mu \mathrm{m}$ GCL length and number of cells $/ 500 \mu \mathrm{m}^{2}$ INL (b) in secretin- and glucagon-treated retinas. The retinoprotective effects of secretin or glucagon were not quantified by the thickness ( $\mu \mathrm{m})$ of retinal layers (a) OPL, INL, and IPL and number of cells $/ 100 \mu \mathrm{m}$ GCL length or the number of cells $/ 500 \mu \mathrm{m}^{2}$ in the INL (b). ONL: outer nuclear layer; OPL: outer plexiform layer; INL: inner nuclear layer; IPL: inner plexiform layer. ${ }^{*} P<0.05$ compared to SHAM retinas.

ameliorate the ischemic damage of the retina (Figures 1(d)$1(\mathrm{i})$ ). In all samples, nuclear layers (ONL, INL) and plexiform layers (OPL, IPL) were reduced, and the cell number in the GCL was significantly less than in sham-operated controls. However, injection with PACAP 6-10 and 4-13 resulted in further reduction of the inner plexiform and nuclear layers (Figures $1(\mathrm{~h})$ and 1(i)). Treatment with members of the PACAP-related peptide superfamily, glucagon and secretin, led to no aggravation or amelioration of the ischemic retinal lesion (Figure 3). These observations were confirmed by morphometric measurements (Figure 4).

PACAP is a member of the secretin/glucagon/VIP superfamily of peptides. PACAP and its receptors occur in the retina [25]. PACAP is thought to play an important role in visual processing, as it is an important cotransmitter in the retinohypothalamic tract that is the major input for the biological clock situated in the suprachiasmatic nucleus [26]. Besides its supposed physiological actions in the retina, PACAP is a well-established retinoprotective peptide.
Endogenous protection is proven by the increased vulnerability of the retina in mice lacking endogenous PACAP in ischemic retinal lesion [2] and in NMDA-induced excitotoxic injury [14]. These observations are further supported by the increased apoptotic activity in retinas injected by the PACAP antagonist PACAP 6-38 [27]. As PACAP is a well-accepted retinoprotective agent in models of different retinal injuries, several studies have attempted to explore the molecular mechanisms explaining this strong retinoprotection [27]. We have shown that PACAP activates the antiapoptotic, while it inhibits the proapoptotic signaling pathways in retinal toxic lesions [27]. In retinal ischemia, we have shown that PACAP administration provides protection by involving Akt, MAPK pathways, and anti-inflammatory actions [28].

Given the very potent actions of PACAP in retinal and other pathologies, there is an urging demand for developing novel analogues and/or fragments to increase the bioavailability and stability of the peptide for future potential 
therapeutic use $[7,8]$. Binding PACAP to other carrier molecules is a possibility to increase its capacity to traverse through membranes and to exert cytoprotective effects [29]. It has also been shown that PACAP is able to pass through cell membranes and convey nonreceptor mediated effects [30]. This has raised the possibility of using PACAP fragments also for supporting transport of other nonpenetrable molecules [31]. Several analogues have also been developed for these purposes $[7,8,32]$. Interestingly, however, a stable analogue did not provide stronger neuroprotection in a stroke model than the natural peptide [33]. Unfortunately, all these efforts do not seem to lead to obtaining of a more effective molecule than the original peptide or to obtaining of a biologically more stable analogue that has the same protective property as PACAP 1-38. The evolutionary pressure on the form of PACAP 1-38 seems to be very strong, as effects found in vertebrates have also been described in invertebrates [7]. Although a lot of pharmacological and receptor binding studies have been performed with fragments/analogues of PACAP, only few studies have tested the biological efficacy of these forms [33]. Since some studies suggest that there are unconventional binding sites, unusual behavior of the peptide forms in some tissues and even implies the existence of unknown receptor splice variants [21, 22, 34]; it is important to test the biological effects of fragments in a system that is well standardized in numerous earlier studies. Our present results show that the shorter fragments of PACAP, as expected, do not provide any neuroprotection. In addition, we can confirm that most of them do not deteriorate the degree of damage either, although some parameters were worsened in cases of PACAP 6-10 and 4-13. This is in accordance with our earlier observations showing that PACAP antagonists PACAP 6-38 and 6-27 lead to aggravated retinal lesion in an MSG-induced retinal injury model [23].

Relatively little is known about other members of the peptide family in the retina. Some other members of this family have been localized in the vertebrate retina and several functions have been attributed to them $[35,36]$. Glucagonergic amacrine cells represent a small subpopulation of the amacrine cells possibly playing a role in the visual processing $[37,38]$. Other studies have shown that glucagon plays a role in eye bulb growth and glucagon is suggested to be an endogenous mediator of emmetropization [39]. An early study has shown that glucagon increases cAMP in chick retinal Muller cells, similar to the actions of VIP [40]. The protective effects of secretin and glucagon in cerebral pathologies have been implicated in a few studies [41-43]. In cerebral ischemia, glucagon has been shown to exert neuroprotective effects in vivo [41]. It has not been investigated so far whether secretin or glucagon is protective in retinal lesions. Based on our present study, neither glucagon nor secretin has protective action in retinal ischemia. Based on our present and previous results, a strong neuroprotective effect is a specific PACAPaction, since VIP could exert its protective effects only in much higher doses, while secretin and glucagon did not have any effects.

Our present results confirm that the natural form of the peptide, PACAP 1-38, is the most effective peptide form in retinal ischemia, and the 38 amino acid form of the peptide cannot be replaced by another fragment or another member of the peptide family that we know of. Our results further support the potent retinoprotective effects of PACAP and call for further studies to establish the future possible clinical introduction of PACAP-related retinoprotective therapeutic approach.

\section{Conflict of Interests}

The authors declare that they have no conflict of interests.

\section{Acknowledgments}

This study was supported by OTKA K104984, K100144, PD 109644, and TAMOP (4.2.2.A-11/1/KONV-2012-0024), the European Union and the State of Hungary, and cofinanced by the European Social Fund in the framework of TAMOP 4.2.4. A/2-11-1-2012-0001 "National Excellence Program," Magyary Zoltan Scholarship, Arimura Foundation, PTE-MTA "Lendulet" Program, and Bolyai Scholarship.

\section{References}

[1] D. Vaudry, A. Falluel-Morel, S. Bourgault et al., "Pituitary adenylate cyclase-activating polypeptide and its receptors: 20 Years after the discovery," Pharmacological Reviews, vol. 61, no. 3, pp. 283-357, 2009.

[2] D. Reglodi, P. Kiss, K. Szabadfi et al., "PACAP is an endogenous protective factor-insights from PACAP-deficient mice," Journal of Molecular Neuroscience, vol. 48, pp. 482-492, 2012.

[3] J. M. Dragich, D. H. Loh, L. M. Wang et al., "The role of the neuropeptides PACAP and VIP in the photic regulation of gene expression in the suprachiasmatic nucleus," European Journal of Neuroscience, vol. 31, no. 5, pp. 864-875, 2010.

[4] E. Borbely, B. Scheich, Z. Helyes et al., "Neuropeptides in learning and memory," Neuropeptides, vol. 47, no. 6, pp. 439450, 2013.

[5] M. Tajiri, A. Hayata-Takano, K. Seiriki et al., "Serotonin 5HT(7) receptor blockade reverses behavioral abnormalities in PACAP-deficient mice and receptor activation promotes neurite extension in primary embryonic hippocampal neurons: therapeutic implications for psychiatric disorders," Journal of Molecular Neuroscience, vol. 48, no. 3, pp. 473-481, 2012.

[6] K. Matsuda, M. Azuma, K. Maruyama, and S. Shioda, "Neuroendocrine control of feeding behavior and psychomotor activity by pituitary adenylate cyclase-activating polypeptide (PACAP) in vertebrates," Obesity Research \& Clinical Practice, vol. 7, no. 1, pp. el-e88, 2013.

[7] S. Bourgault, D. Vaudry, A. Dejda, N. D. Doan, H. Vaudry, and A. Fournier, "Pituitary adenylate cyclase-activating polypeptide: focus on structure-activity relationships of a neuroprotective peptide," Current Medicinal Chemistry, vol. 16, no. 33, pp. 4462-4480, 2009.

[8] S. Bourgault, D. Chatenet, O. Wurtz et al., "Strategies to convert PACAP from a hypophysiotropic neurohormone into a neuroprotective drug," Current Pharmaceutical Design, vol. 17, no. 10, pp. 1002-1024, 2011.

[9] A. Dejda, S. Bourgault, N. D. Doan et al., "Identification by photoaffinity labeling of the extracellular N-terminal domain of PAC1 receptor as the major binding site for PACAP," Biochimie, vol. 93, no. 4, pp. 669-677, 2011. 
[10] Y. Yan, X. Zhou, Z. Pan, J. Ma, J. A. Waschek, and E. DiCiccoBloom, "Pro- and anti-mitogenic actions of pituitary adenylate cyclase-activating polypeptide in developing cerebral cortex: potential mediation by developmental switch of PAC1 receptor mRNA isoforms," Journal of Neuroscience, vol. 33, no. 9, pp. 3865-3878, 2013.

[11] K. Shoge, H. K. Mishima, T. Saitoh et al., "Attenuation by PACAP of glutamate-induced neurotoxicity in cultured retinal neurons," Brain Research, vol. 839, no. 1, pp. 66-73, 1999.

[12] E. Fabian, D. Reglodi, L. Mester et al., "Effects of PACAP on intracellular signaling pathways in human retinal pigment epithelial cells exposed to oxidative stress," Journal of Molecular Neuroscience, vol. 48, pp. 493-500, 2012.

[13] T. Atlasz, K. Szabadfi, P. Kiss et al., "PACAP-mediated neuroprotection of neurochemically identified cell types in MSGinduced retinal degeneration," Journal of Molecular Neuroscience, vol. 36, no. 1-3, pp. 97-104, 2008.

[14] K. Endo, T. Nakamachi, T. Seki et al., "Neuroprotective effect of PACAP against NMDA-induced retinal damage in the mouse," Journal of Molecular Neuroscience, vol. 43, no. 1, pp. 22-29, 2011.

[15] T. Atlasz, K. Szabadfi, P. Kiss et al., "Effects of PACAP in UV-A radiation-induced retinal degeneration models in rats," Journal of Molecular Neuroscience, vol. 43, no. 1, pp. 51-57, 2011.

[16] T. Seki, H. Itoh, T. Nakamachi, and S. Shioda, "Suppression of ganglion cell death by PACAP following optic nerve transection in the rat," Journal of Molecular Neuroscience, vol. 36, no. 1-3, pp. 57-60, 2008.

[17] K. Szabadfi, A. Szabo, P. Kiss et al., "PACAP promotes neuron survival in early experimental diabetic retinopathy," Neurochemistry International, vol. 64, pp. 84-91, 2014.

[18] T. Seki, H. Itoh, T. Nakamachi et al., "Suppression of rat retinal ganglion cell death by PACAP following transient ischemia induced by high intraocular pressure," Journal of Molecular Neuroscience, vol. 43, no. 1, pp. 30-34, 2011.

[19] T. Atlasz, K. Szabadfi, P. Kiss et al., "Evaluation of the protective effects of PACAP with cell-specific markers in ischemiainduced retinal degeneration," Brain Research Bulletin, vol. 81, no. 4-5, pp. 497-504, 2010.

[20] T. Atlasz, K. Szabadfi, P. Kiss et al., "Pituitary adenylate cyclase activating polypeptide in the retina: focus on the retinoprotective effects," Annals of the New York Academy of Sciences, vol. 1200, pp. 128-139, 2010.

[21] D. Reglodi, R. Borzsei, T. Bagoly et al., "Agonistic behavior of PACAP6-38 on sensory nerve terminals and cytotrophoblast cells," Journal of Molecular Neuroscience, vol. 36, no. 1-3, pp. 270-278, 2008.

[22] C. S. Walker, T. Sundrum, and D. L. Hay, "PACAP receptor pharmacology and agonist bias: analysis in primary neurons and glia from the trigeminal ganglia and transfected cells," British Journal of Pharmacology, vol. 171, pp. 1521-1533, 2014.

[23] T. Atlasz, K. Szabadfi, D. Reglodi et al., "Effects of pituitary adenylate cyclase activating polypeptide and its fragments on retinal degeneration induced by neonatal monosodium glutamate treatment," Annals of the New York Academy of Sciences, vol. 1163, pp. 348-352, 2009.

[24] K. Szabadfi, B. Danyadi, P. Kiss et al., "Protective effects of Vasoactive Intestinal Peptide (VIP) in ischemic retinal degeneration," Journal of Molecular Neuroscience, vol. 48, pp. 501-507, 2012.
[25] T. Nakamachi, A. Matkovits, T. Seki, and S. Shioda, "Distribution and protective function of pituitary adenylate cyclaseactivating polypeptide in the retina," Endocrinolology (Lausanne), vol. 3, p. 145, 2012.

[26] C. Kawaguchi, Y. Isojima, N. Shintani et al., "PACAP-deficient mice exhibit light parameter-dependent abnormalities on nonvisual photoreception and early activity onset," PLOS ONE, vol. 5, no. 2, article e9286, 2010.

[27] B. Racz, F. Gallyas Jr., P. Kiss et al., "The neuroprotective effects of PACAP in monosodium glutamate-induced retinal lesion involve inhibition of proapoptotic signaling pathways," Regulatory Peptides, vol. 137, no. 1-2, pp. 20-26, 2006.

[28] A. Szabo, B. Danyadi, E. Bognar et al., "Effect of PACAP on MAP kinases, Akt and cytokine expressions in rat retinal hypoperfusion," Neuroscience Letters, vol. 523, no. 2, pp. 93-98, 2012.

[29] R. Yu, X. Guo, L. Huang, Z. Zeng, and H. Zhang, "The novel peptide PACAP-TAT with enhanced traversing ability attenuates the severe lung injury induced by repeated smoke inhalation," Peptides, vol. 38, no. 1, pp. 142-149, 2012.

[30] N.-D. Doan, D. Chatenet, M. Létourneau, H. Vaudry, D. Vaudry, and A. Fournier, "Receptor-independent cellular uptake of pituitary adenylate cyclase-activating polypeptide," Biochimica et Biophysica Acta, vol. 1823, no. 4, pp. 940-949, 2012.

[31] N. D. Doan, M. Letourneau, D. Vaudry et al., "Design and characterization of novel cell-penetrating peptides from pituitary adenylate cyclase-activating polypeptide," Journal of Controlled Release, vol. 163, no. 2, pp. 256-265, 2012.

[32] N.-D. Doan, S. Bourgault, A. Dejda et al., "Design and in vitro characterization of PAC1/VPAC1-selective agonists with potent neuroprotective effects," Biochemical Pharmacology, vol. 81, no. 4, pp. 552-561, 2011.

[33] A. Dejda, T. Seaborn, S. Bourgault et al., "PACAP and a novel stable analog protect rat brain from ischemia: insight into the mechanisms of action," Peptides, vol. 32, no. 6, pp. 1207-1216, 2011.

[34] J. M. Muller, C. Debaigt, S. Goursaud et al., "Unconventional binding sites and receptors for VIP and related peptides PACAP and PHI/PHM: an update," Peptides, vol. 28, no. 9, pp. 1655$1666,2007$.

[35] U. Mathis and F. Schaeffel, "Glucagon-related peptides in the mouse retina and the effects of deprivation of form vision," Graefe's Archive for Clinical and Experimental Ophthalmology, vol. 245, no. 2, pp. 267-275, 2007.

[36] A. P. Swedlund and S. A. Rosenzweig, "Characterization of vasoactive intestinal peptide receptors in retina," Experimental Eye Research, vol. 51, no. 3, pp. 317-323, 1990.

[37] W. D. Eldred, J. Ammermuller, J. Schechner, U. D. Behrens, and R. Weiler, "Quantitative anatomy, synaptic connectivity and physiology of amacrine cells with glucagon-like immunoreactivity in the turtle retina," Journal of Neurocytology, vol. 25, no. 5, pp. 347-364, 1996.

[38] E. Diedrich and F. Schaeffel, "Spatial resolution, contrast sensitivity, and sensitivity to defocus of chicken retinal ganglion cells in vitro," Visual Neuroscience, vol. 26, no. 5-6, pp. 467-476, 2009.

[39] K. A. Vessey, K. A. Lencses, D. A. Rushforth, V. J. Hruby, and W. K. Stell, "Glucagon receptor agonists and antagonists affect the growth of the chick eye: a role for glucagonergic regulation of emmetropization?" Investigative Ophthalmology and Visual Science, vol. 46, no. 11, pp. 3922-3931, 2005.

[40] S. W. M. Koh, A. Kyritis, and G. J. Chader, "Interaction of neuropeptides and cultured glial (Muller) cells of the chick 
retina: elevation of intracellular cyclic AMP by vasoactive intestinal peptide and glucagon," Journal of Neurochemistry, vol. 43, no. 1, pp. 199-203, 1984.

[41] R. A. Fanne, T. Nassar, S. N. Heyman, N. Hijazi, and A. A. R. Higazi, "Insulin and glucagon share the same mechanism of neuroprotection in diabetic rats: role of glutamate," American Journal of Physiology-Regulatory Integrative and Comparative Physiology, vol. 301, no. 3, pp. R668-R673, 2011.

[42] R. Abu Fanne, T. Nassar, A. Mazuz et al., "Neuroprotection by glucagon: role of gluconeogenesis. Laboratory investigation," Journal of Neurosurgery, vol. 114, no. 1, pp. 85-91, 2011.

[43] I. P. Y. Lam, F. K. Y. Siu, J. Y. S. Chu, and B. K. C. Chow, "Multiple actions of secretin in the human body," International Review of Cytology, vol. 265, pp. 159-190, 2008. 


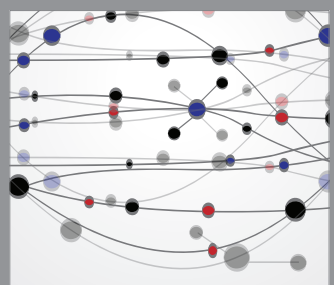

The Scientific World Journal
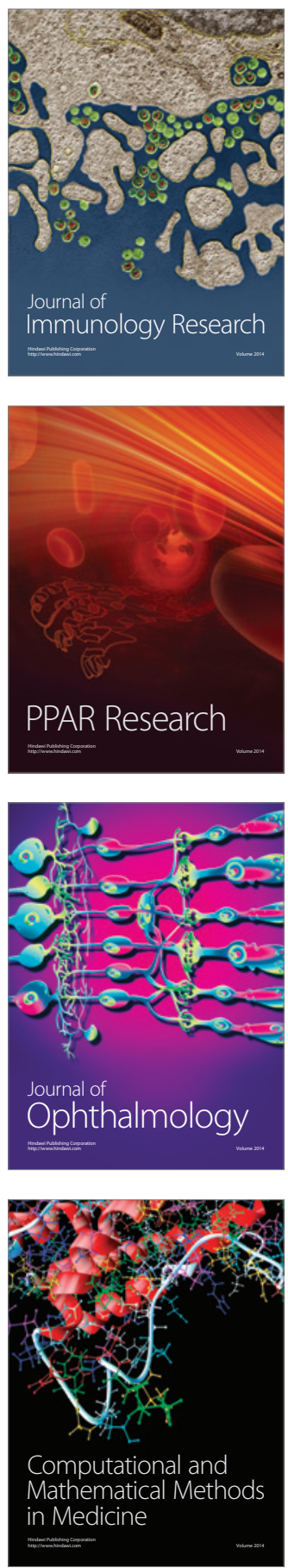

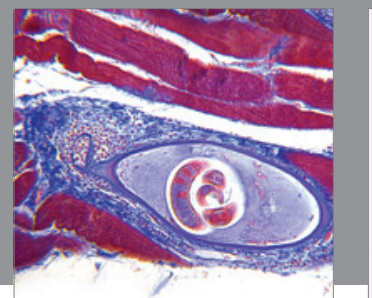

Gastroenterology

Research and Practice
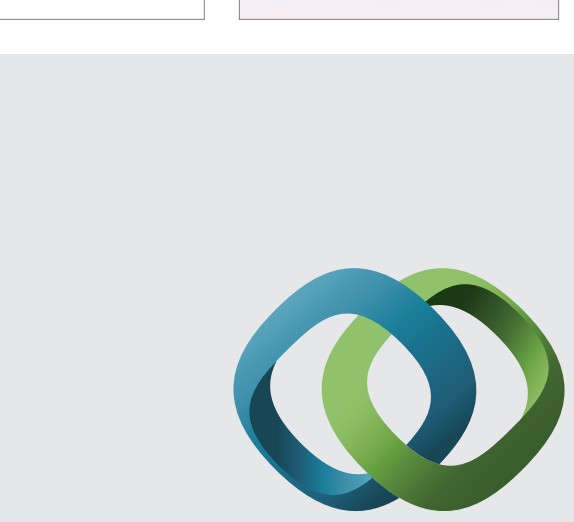

\section{Hindawi}

Submit your manuscripts at

http://www.hindawi.com
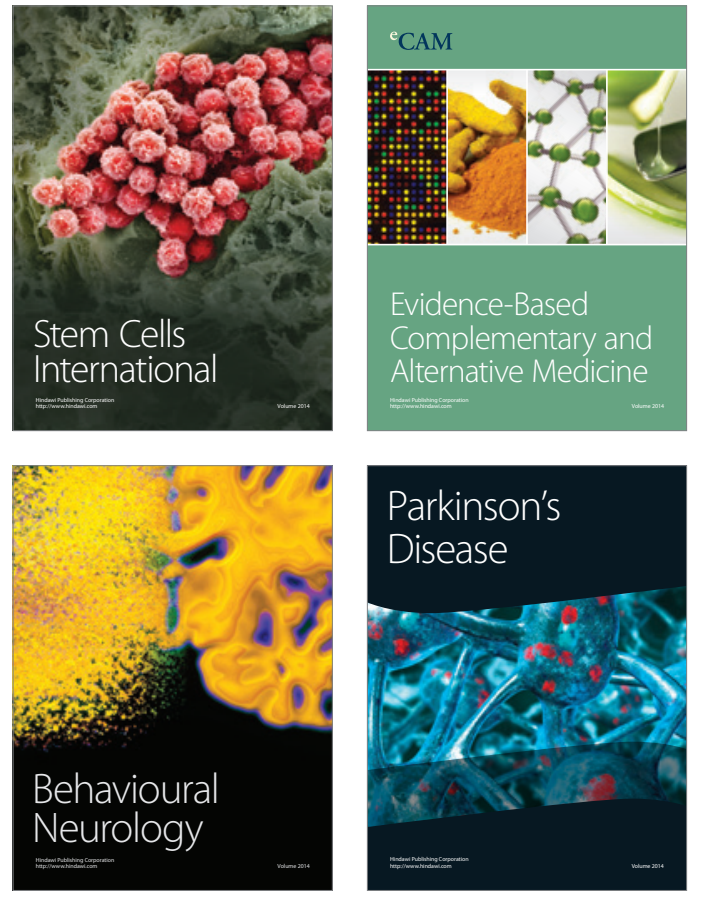
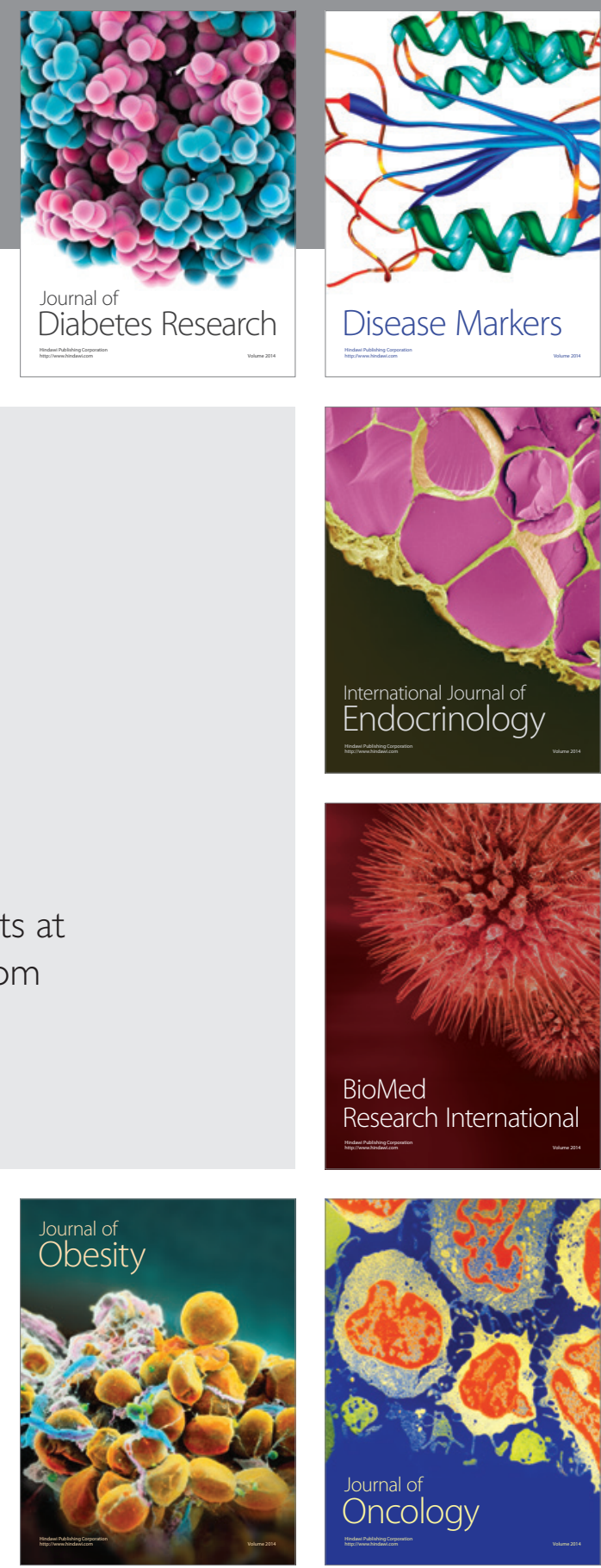

Disease Markers
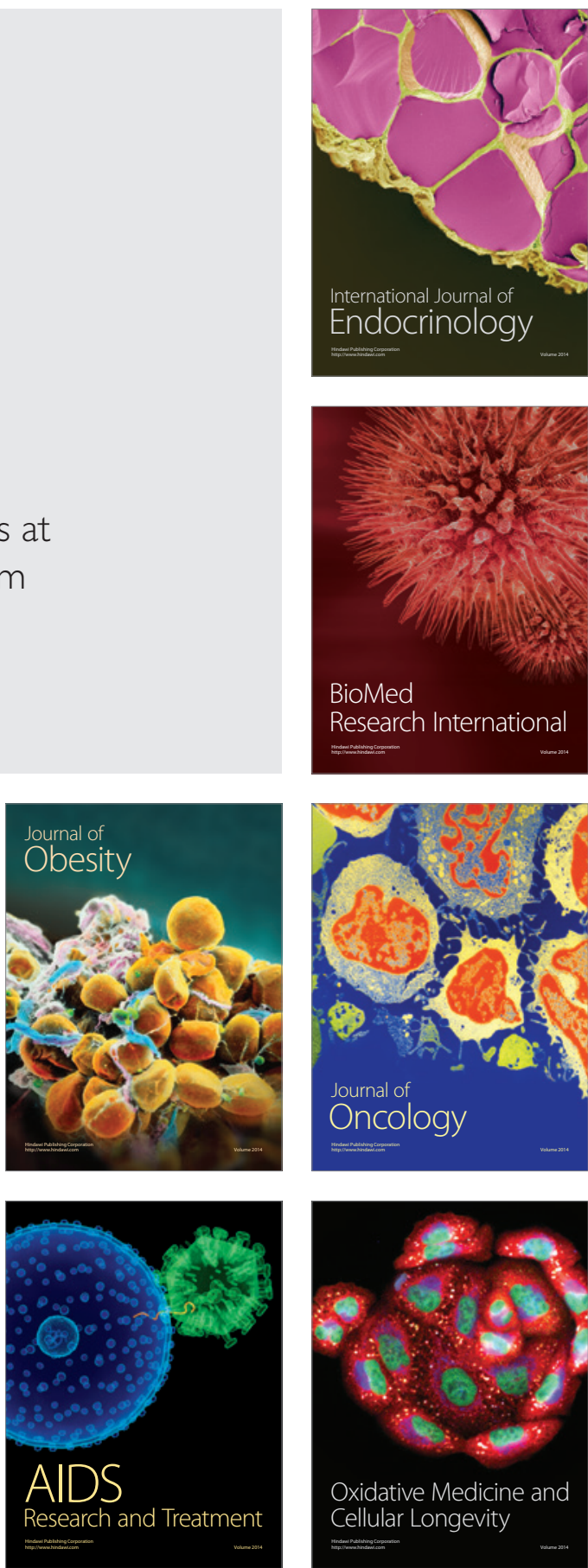